\title{
Biologic: A Double Edged Sword - A Case of Etanercept Induced Alopecia Areata in a Patient with Psoriasis Vulgaris
}

\author{
Sundeep Chowdhry ${ }^{*}$, Priya $^{1}$, Paschal D’Souza ${ }^{2}$, Rishi Goel ${ }^{2}$ and Rohini Soni ${ }^{1}$ \\ ${ }^{1}$ Department of Dermatology, Venerology and Leprology, Room 107 First Floor, ESI-PGIMSR, Basaidarapur, New Delhi \\ ${ }^{2}$ Department of Dermatology, Venerology and Leprology, Room 104 First Floor, ESI-PGIMSR, Basaidarapur, New Delhi
}

Received: 17 November, 2017; Accepted: 28 December, 2017; Published: 18 January, 2018

*Corresponding author: Sundeep Chowdhry, Department of Dermatology, Venerology and Leprology, Room 107 First Floor, ESI-PGIMSR, Basaidarapur, New Delhi, Email: suncutis@gmail.com

\begin{abstract}
Psoriasis vulgaris is a chronic inflammatory disorder which can only be controlled with medications but not cured. Various modalities are available for the management of same with varying efficacy and adverse effects. Biologics, though not new, have changed the concept of treatment of psoriasis. With beneficial effects, they also have adverse effects which are being identified more and more with passing time.
\end{abstract}

\section{Introduction}

Biologics have revolutionized the treatment modalities in various diseases specially those having the autoimmune diathesis and have become an important treatment alternative for systemic and cutaneous auto-immune diseases. These agents are target specific and mainly act against cytokines, B cells, and costimulation molecules. Owing to their ability to target proteins in a more specific manner, biologics have comparatively lower risks of systemic side-effects, thus having considerable advantage over the conventional immune-modulator drugs. The goal of biologics is to weaken or immobilize those features of the immune system that trigger autoimmune diseases without the risk of broadly weakening the immune system as encountered with other immunosuppressant [1]. The increased use of biologic drugs over the period of time have revealed new adverse effects of these pharmaceutical agents. The cutaneous reactions of biologics described so far usually include eczema, erythema, urticaria, lupuslike syndrome and paradoxically psoriasis itself [2].

Alopecia Areata (AA) is a non scarring patchy or diffuse loss of hair on the scalp or any hair bearing area of the body. It affects approximately $1.7 \%$ of the overall population, with no sex preponderance, predominantly found in females as they are more likely to seek treatment for this ailment than their counterparts. The etiology is unknown but there are indications of T-cellmediated autoimmune process directed against an unknown auto antigen of the hair follicle. $\mathrm{T}$ lymphocytes that have been shown to be oligoclonal and auto-reactive are predominantly present in the peribulbous inflammatory infiltrate [3].
The hair follicles are reversibly damaged mainly by cytotoxic $\mathrm{T}$ lymphocytes and cytokines (in-terferon- $\gamma$, interleukin-2, and interleukin-15 receptor $\beta$ ); which eventually results in falling out of the hair from the follicle [4]. Tumor Necrosis Factor- Alpha (TNF Alpha) is a pro-inflammatory cytokine and acts as an acute phase reactant which is produced mainly by activated macrophages and other cells like CD4+ lymphocytes, neutrophils, eosinophils, etc. Blockade of TNF alpha re-lease by blocking the receptors and/or blocking the free circulating TNF alpha may be helpful in preventing the damage to the hair follicle caused by cytotoxic lymphocytes. Nails are involved in $10-66 \%$ of all AA cases. Nails can be affected before or after hair loss and nail involvement may persist even after treatment and hair re growth $[5,6]$.

It is worthwhile to highlight the paradoxical effect of the molecule wherein on one hand it leads to improvement of the disease in a recalcitrant case and on the other hand the same molecule shows the opposite effect by either deteriorating the preexisting condition or inducing new symptomatology as experienced in our patient.

\section{Case report}

A 42 years old male, known case of Psoriasis vulgaris for the last 12 years presented with chief complaints of generalized scaly plaques involving approximately $50 \%$ of body surface area. The patient was non alcoholic, non smoker, with no history of other autoimmune disease, no history of atopy and no family history of alopecia. Patient's thyroid profile was within normal limits. Patient was previously treated with oral methotrexate, cyclosporine, acitretin, topical steroids, topical keratolytics, topical vitamin D analogues and phototherapy (NB-UVB) with minimal response. The patient did not attain satisfactory response with the above treatment modalities and the disease being progressive in nature resulted in further deterioration and adversely affected his quality of life. In view of the above course of events he was planned for Etanercept therapy. Complete biochemical evaluation prior to starting a biological drug was carried out including complete blood count, liver function test, kidney function test, 
blood sugar, viral serologies (Hepatitis and HIV), quantiferon gold test and ECG. All investigations were within normal limits and consequently the therapy was initiated with Injection Etanercept as per the prescribed schedule [7]. He was initially treated with 50mg Injection Etanercept given subcutaneously twice weekly for 12 weeks, followed by once weekly injection for further 12 weeks. The patient started improving 4 weeks after receiving the first dose of Inj Etanercept and reported marked improvement with the therapy towards the end of the treatment schedule. After 20 weeks the patient started complaining of decreased hair density over the eyebrows and moustache (Figure 1). Patches of alopecia areata were clinically visible after 22 weeks of initiation of Inj Etanercept. Since only two more injections were left to complete the maintenance dose regimen of Inj Etanercept, it was considered prudent to continue Etanercept for a period of further two weeks. In the meantime patient was co-prescribed oral Biotin and oral Iron supplements. Inj. Etanercept was discontinued after the completion of said regimen and the patient was continued on oral medications. After 2 weeks of discontinuation of Etanercept therapy, the patch of alopecia became non progressive and improved within 12 weeks (Figure 2).

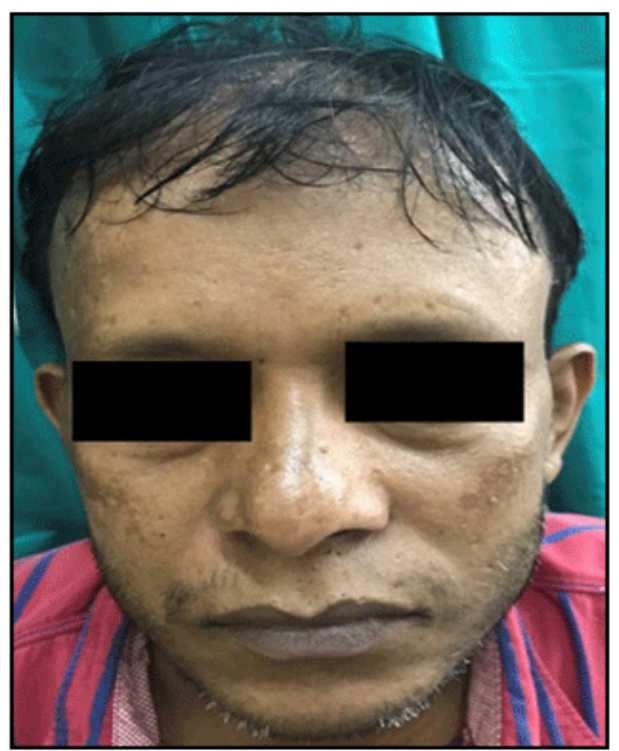

Figure 1: Alopecia areata (beard) after inj. Etanercept

\section{Discussion}

Although on one hand biologics have emerged as an invincible spearhead against numerous autoimmune disorders which adversely impact the quality of life but on the other hand the side effect of these agents has shown a surge all around the globe. Common adverse effects of biologics include hypersensitivity reaction, higher rates of concomitant severe infections, reactivation of tuberculosis etc, the latter being more common in the developing countries [8]. Apart from the commonly advocated adverse effects, there are certain other side effects which are either less known or are under reported. Occurrence of alopecia areata with TNF $\alpha$ antagonists (Etanercept) is such

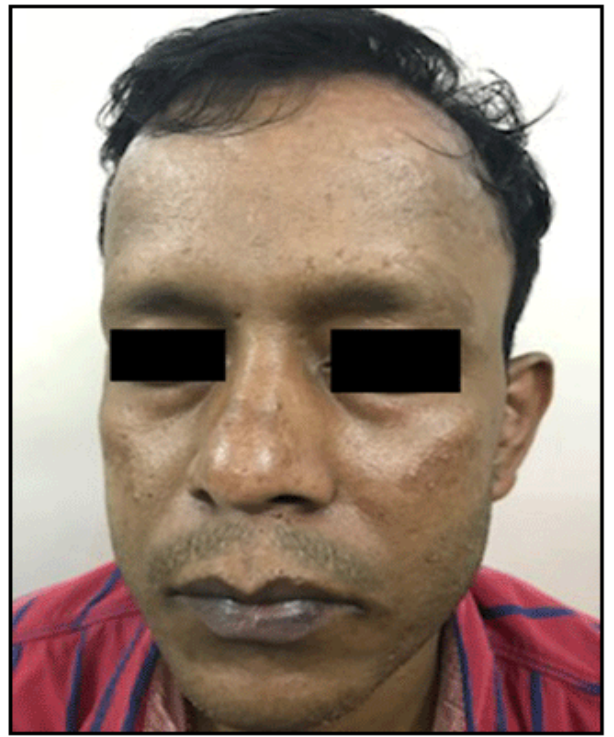

Figure 2: Alopecia areata (beard) resolved after discontinuation of inj. Etanercept

an adverse effect which has been reported by few authors in past two decades [9]. Riebiero LBP, et al. reported two cases of TNF alpha antagonist Infliximab induced Alopecia areata in patients of Crohn's disease where the patient developed AA after 3 years of Infliximab therapy and the other developed AA after 4 months of the initiation of therapy [10].

Lindsay, et al. have reported 34 cases of AA consequent to initiation of TNF alpha antagonists till 2013 [11].

In our case the time elapsed after the introduction of the antiTNF alpha agent Etanercept to the development of AA was 4.5 months which is similar to the case study by Le Bidre, et al. [12].

Improvement in alopecia even without treatment on cessation of the Etanercept strongly high-lights the association between the two entities. However other causes of alopecia areata cannot be ruled out completely in our patient on the basis of insignificant personal or family history as it is known that genetic predisposition and environmental factors play a significant role in the etiopathogenesis of alopecia areata. The possible mechanism of development of alopecia areata with TNF $\alpha$ antagonists has not been well understood till date.

Kasumagic E, et al. conducted a study in 36 patients of AA with 20 Controls in which they found that the patients with AA had significantly increased TNF alpha levels in comparison to the levels found in Controls [13]. Working on the same principle Gorcey, et al. had achieved improvement in a case of recalcitrant alopecia universalis with the use of TNF alpha antagonist [14].

The case has been mentioned to highlight the astonishing paradoxical effect of TNF alpha antagonists that on one hand there are cases which have been reported to be as alopecia areata occur-ring after administration of TNF $\alpha$ antagonist but on the other hand there are case reports where-in severe alopecia universalis has improved with the use of TNF $\alpha$ antagonists [14]. 


\section{References}

1. Feldmann M, Steinman L. Design of effective immunotherapy for human autoimmunity. Nature. 2005;435(7042):612-619.

2. Iborra M, Beltran B, Bastida G, Aguas M, Nos P. Infliximab and adalimumab-induced psori-asis in Crohn's disease: A paradoxical side effect. J Crohns Colitis. 2011;5(2):157-161. doi: 10.1016/j. crohns.2010.11.001

3. Hordinsky M, Ericson M. Autoimmunity: alopecia areata. J Investig Dermatol Symp Proc. 2004;9(1):73-78.

4. Gilhar A, Etzioni A, Paus R. Alopecia areata. N Engl J Med. 2012;366(16):1515-1525. doi: 10.1056/NEJMra1103442

5. Kasumagic-Halilovic E, Prohic A. Nail changes in alopecia areata: frequency and clinical presentation. J Eur Acad Dermatol Venereol 2009;23(2):240-241. doi: 10.1111/j.1468-3083.2008.02830.x

6. Tosti A, Pazzaglia M, Venturi M, Chiacchio NC Jr. Severe onycholysis in a card illusionist with alopecia areata universalis. Int J Trichology. 2013;5(2):81-82. doi: 10.4103/0974-7753.122966

7. Segaert S. Etanercept, improved dosage schedules and combinations in the treatment of pso-riasis: an update. J Inflamm Res. 2009;2:29-36.
8. Navarra SV, Tang B, Lu L, Lin HY, Mok CC, Asavatanabodee P, et al. Risk of tuberculosis with anti-tumor necrosis factor- $\alpha$ ther-apy: substantially higher number of patients at risk in Asia. Int J Rheum Dis. 2014;17(3):291-298. doi: 10.1111/1756-185X.12188

9. Tosti A, Pazzaglia M, Starace M, Bellavista S, Vincenzi C, Tonelli G. Alopecia Areata During Treatment With Biologic Agents. Arch Dermatol. 2006;142(12):1653-1654.

10.Riebiero LB, Rego JC, Estrada BD, Bastos PR, Maceira JM, Sodre CT Alopecia secondary to anti-tumor necrosis factor-alpha therapy. An Bras Dermatol. 2015;90(2):232-235. doi: 10.1590/abd18064841.20153084

11.Lindsay SF, Tosti A. Hair loss induced by tumor necrosis factor alpha inhibitors. J Clin Investigat Dermatol. 2013;1(1):6

12.Le Bidre E, Chaby G, Martin L, Perrussel M, Sassolas B, Sigal ML, et al. Ann Dermatol Venereol. 2011;138(4):285-293. doi: 10.1016/j. annder.2011.01.047

13.Kasumagic-Halilovic E, Prohic A, Cavaljuga S. Tumor necrosis factor-alpha in patients with alopecia areata. Indian J Dermatol. 2011;56(5):494-496. doi: 10.4103/0019-5154.87124

14.Gorcey L, Gordon Spratt EA, Leger MC. Alopecia universalis successfully Treated With adalimumab. JAMA Dermatol. 2014;150(12):1341-1344. 